\title{
ON A REFLECTIVE SUBCATEGORY OF THE CATEGORY OF ALL TOPOLOGICAL SPACES
}

\author{
BY \\ LADISLAV SKULA
}

1. Introduction. In his paper [4] J. F. Kennison gives three types of full reflective subcategories of the category of all topological spaces called simple, identifying and embedding and notes that he does not know whether these three types include all of the full reflective subcategories of all topological spaces.

In my paper I describe another type called b-embedding not mentioned in [4] and I prove that if $P$ is a full reflective (replete) subcategory of the category of all topological spaces containing at least one non- $T_{1}$-space, then $P$ is a subcategory of one of the above-mentioned types (Theorem 3.5).

For the understanding of my paper the basic knowledge of topology according to Kelley's General topology [3] is required. Thus a topological space will be an ordered pair $(A, L)$, where $L$ is the family of all open subsets of the underlying set $A$. (If there is no danger of confusion only $A$ will denote the topological space.) The closure of $M \subset A$ will be denoted by $\mathrm{cl}_{(A, L)} M=\mathrm{cl}_{A} M=\mathrm{cl} M ; T$ will mean the category of all topological spaces with morphisms as continuous maps, and $T_{0}$ (resp. $T_{1}$ ) the full subcategory of $T_{0}$-spaces (resp. $T_{1}$-spaces). The topological product of topological spaces $X_{\iota}, \iota \in \mathscr{I} \neq \varnothing$ will be denoted by $\prod X_{\iota}(\iota \in \mathscr{I})$.

Further I presuppose the knowledge of Kennison's paper [4]. Recall that if $F$ is a reflector of a category $A$ in a category $B$, then for $X \in A e_{X}$ will denote the reflection map or the front adjunction map $(\in \operatorname{Hom}(X, F(X))$ with the property. $e_{Y} f=F(f) e_{X}$ for all $f \in \operatorname{Hom}(X, Y)$. By a topological property there is meant a full subcategory of $T$ which is closed under the formation of equivalent (=homeomorphic) objects (full replete subcategory of $T$ ). A productive (resp. a closed hereditary) topological property is such that it is closed under the formation of product spaces (resp. closed subspaces). The full subcategory $P$ of $T$ is simple (resp. identifying, or embedding) iff there exists a reflector $F: T \rightarrow P$. such that $e_{X}: X \rightarrow F(X)$ is one-to-one and onto for all $X \in T$ (resp. $e_{X}$ is onto, or $P$ contains only Hausdorff spaces and $e_{X}$ is onto a dense subset).

From the Theorem on p. 878 in Notes [1] follows immediately:

1.1. Let $P$ be a topological property which is reflective in $T$. Then $P$ is productive.

2. $b$-topology.

2.1. Let $(A, L) \in T$. For $X \subset A$ let $b X=b_{A} X=b_{(A, L)} X$ denote the set of all points

Received by the editors July 24, 1968. 
$p \in A$ with the following property: there exist no $G_{1}, G_{2} \in L$ such that $p \in G_{2}-G_{1}$ and $G_{2} \cap X=G_{1} \cap X$. For $X, Y \subset A$ the following assertion holds:

(a) $X \subset b X \subset \mathrm{cl} X$,

(b) $X \subset Y \Rightarrow b X \subset b Y$,

(c) $b X=b b X$,

(d) $b(X \cup Y)=b X \cup b Y$.

Proof. The assertions (a) and (b) are clear. Assertion (c) follows in the following manner: according to (a) and (b) there holds $b X \subset b b X$. Let $p \in A-b X$. Then there exist $G_{1}, G_{2} \in L, p \in G_{2}-G_{1}, G_{2} \cap X=G_{1} \cap X$. Hence for $x \in\left(G_{2}-G_{1}\right)$ $\cup\left(G_{1}-G_{2}\right)$ there holds $x \notin b X$. Thus $G_{2} \cap b X=G_{1} \cap b X$, therefore $p \notin b b X$.

Assertion (d): There holds $X \subset X \cup Y, Y \subset X \cup Y$, whence according to (b), $b X \cup b Y \subset b(X \cup Y)$. Let $p \in A-b X \cup b Y$; then there exist $G_{1}, G_{2}, G_{1}^{\prime}, G_{2}^{\prime} \in L$ such that $G_{1} \cap X=G_{2} \cap X, G_{1}^{\prime} \cap Y=G_{2}^{\prime} \cap Y, p \in G_{2}-G_{1}$ and $p \in G_{2}^{\prime}-G_{1}^{\prime}$. Put $H_{2}=G_{2} \cap G_{2}^{\prime}, \quad H_{1}=\left(G_{1} \cup G_{1}^{\prime}\right) \cap H_{2}$. Then $H_{1}, H_{2} \in L, \quad H_{1} \cap(X \cup Y)=H_{2}$ $\cap(X \cup Y), p \in H_{2}-H_{1}$. Hence $p \notin b(X \cup Y)$.

On the space $A$ a new topology, which we shall call b-topology (of the topological space $A$ ), is therefore defined as a closure by means of the operator $b$. To the topological concepts belonging to this $b$-topology we shall give the usual names preceded by $b$, e.g. $b$-closed set, $b$-dense set, etc.

2.2. Let $(A, L) \in T, \mathfrak{A}=\left\{\left(G_{2}-G_{1}\right) \cup\left(G_{1}-G_{2}\right) \mid G_{1}, G_{2} \in L\right\}$. Then $\mathfrak{A}$ is a base of open sets with regard to the b-topology of the space $(A, L)$.

Proof. I. Let $G_{1}, G_{2} \in L, M=A-\left(G_{2}-G_{1}\right) \cup\left(G_{1}-G_{2}\right)$. Then $G_{2} \cap M=G_{1} \cap M$, whence $b M=M$, thus $\left(G_{2}-G_{1}\right) \cup\left(G_{1}-G_{2}\right)$ is a $b$-open set.

II. Let $x \in A$, $U$ be a $b$-open set and $x \in U$. Then $x \notin b(A-U)$, hence there exist $G_{1}, G_{2} \in L, x \in G_{2}-G_{1}, G_{2} \cap(A-U)=G_{1} \cap(A-U)$. Then $\left[\left(G_{2}-G_{1}\right) \cup\left(G_{1}-G_{2}\right)\right]$ $\cap b(A-U)=\varnothing$, whence $\left(G_{2}-G_{1}\right) \cup\left(G_{1}-G_{2}\right) \subset U$. Q.E.D.

Corollary. Let $G \in L$. Then $b G=G$.

Proof. Putting $G_{1}=G, G_{2}=A$, then according to $2.2, A-G$ is $b$-open, therefore $G$ is $b$-closed.

REMARK. The base $\mathfrak{A}$ however is not generally the system of all $b$-open sets of the space $(A, L)$.

For example: let $A$ be the set of all positive integers, for $n \in A$ put $X_{n}=\{m \in A \mid m$ $\geqq n\}, L=\left\{X_{n} \mid n \in A\right\} \cup\{\varnothing\}$. Then $(A, L) \in T$ and the $b$-topology of the space $(A, L)$ is discrete. But $\left.\mathfrak{A}=L \cup\left\{X_{n}-X_{m} \mid n, m \in A\right\} \neq 2^{A} .{ }^{1}\right)$

2.3. Definition. A topological space $B$ is called a $b$-hull of a space $A$ if $A$ is a subspace of $B$ and $b A=B$; in other words $A$ is $b$-dense in $B$.

2.4. Let $(B, K) \in T_{0}$ be a b-hull of a space $(A, L)$. Then card $B \leqq \exp \exp$ card $A .\left({ }^{2}\right)$ Proof. Let $b_{1}, b_{2} \in B, b_{1} \neq b_{2}$. Put $\mathfrak{A}_{i}=\{U \cap A \mid U$ is a neighborhood of the point $\left.b_{i}\right\}$ for $i=1,2$. Then $\mathfrak{A}_{1}, \mathfrak{A}_{2}$ are filters on the set $A$. We can suppose that there

(1) $2^{A}$ denotes the system of all subsets of the set $A$.

${ }^{(2)}$ The symbol card $M$ denotes the cardinal number of the set $M$ and exp card $M=\operatorname{card} 2^{M}$. 
exists $U \in K, b_{2} \in U, b_{1} \notin U$. It holds that $U \cap A \in \mathfrak{A}_{2}$. If $U \cap A \in \mathfrak{A}_{1}$, then there exists $V \in K, b_{1} \in V, V \cap A \subset U \cap A$. Put $V \cup U=W$. Then $W \in K, W \cap A$ $=U \cap A, b_{1} \in W-U$, therefore $b_{1} \notin b A$, which is a contradiction.

Hence $U \cap A \in \mathfrak{A}_{1}$, therefore $\mathfrak{A}_{1} \neq \mathfrak{A}_{2}$. Then card $B \leqq$ card $F(A)$, where $F(A)$ is the set of all filters on the set $A$. Since card $F(A)=\exp \exp$ card $A$, it holds that card $B \leqq \exp \exp$ card $A$.

2.5. Let $A, B \in T, C \in T_{0}, B$ be a b-hull of $A, f \in \operatorname{Hom}_{T}(A, C)$. Then there exists at most one continuous extension of $f$ on $B$. If $C$ is an arbitrary topological space and $f^{*}$ any continuous extension of $f$ on $B$, then $f^{*}(B) \subset b f(A)$.

Proof. I. Let $g, h$ be two different continuous extensions of $f$ on $B$. Then there exists $y \in B$ such that $g(y) \neq h(y)$. We can suppose that there exists an open set $G^{\prime}$ of the space $C$ such that $g(y) \in G^{\prime}, h(y) \in G^{\prime}$. Put $G_{1}=h^{-1}\left(G^{\prime}\right), G_{2}=g^{-1}\left(G^{\prime}\right)$. Then $G_{1}, G_{2}$ are open sets of the space $B$ and $G_{1} \cap A=G_{2} \cap A, y \in G_{2}-G_{1}$, which is a contradiction.

II. Let $f^{*}$ be the continuous extension of $f$ on $B, x \in B, G_{1}^{\prime}, G_{2}^{\prime}$ be open sets of the space $C, G_{1}^{\prime} \cap f(A)=G_{2}^{\prime} \cap f(A), f^{*}(x) \in G_{2}^{\prime}-G_{1}^{\prime}$. Then $G_{1}=f^{*-1}\left(G_{1}^{\prime}\right), G_{2}$ $=f^{*-1}\left(G_{2}^{\prime}\right)$ are open sets of the space $B, G_{1} \cap A=G_{2} \cap A$ and $x \in G_{2}-G_{1}$, which is a contradiction. Thus $f^{*}(B) \subset b f(A)$.

REMARK. After writing this paper I learned that $\mathrm{S}$. Baron obtained the first part of 2.5 in [5]. S. Baron defined equivalently $p \in b X$ iff $p \in \operatorname{cl}(X \cap \operatorname{cl}\{p\})$.

2.6 Let $X, Y \in T_{0}, Y$ be a b-hull of $X, f \in \operatorname{Hom}_{T}(Y, X), f \mid X=i_{X} \cdot\left({ }^{3}\right)$ Then $Y=X$.

Proof. Let $j: X \rightarrow Y$ be the identity embedding of $X$ into $Y$. Then $f j=i_{X}$. Clearly $j f j=j$ and $i_{Y} j=j$ so $j f$ and $i_{Y}$ are continuous extensions of $j$ on $Y$. According to 2.5 $j f=i_{Y}$, which implies $X=Y$.

2.7. Let $\mathscr{I} \neq \varnothing$ be a set, $X_{\iota} \in T$ and $M_{\iota} \subset X_{\iota}$ be a b-closed set for each $\iota \in \mathscr{I}$, $X=\prod X_{\imath}(\iota \in \mathscr{I}), M=\{x \in X \mid x(\iota) \in M$, for each $\iota \in \mathscr{I}\}$. Then $M$ is $b$-closed in $X$.

Proof. Let $p$ be the $\iota$ th projection map $X \rightarrow X_{\iota}$ for all $\iota \in \mathscr{I}$. According to 2.5 $p_{\iota} b M \subset b p_{\iota}(M)=b M_{\iota}=M_{\iota}$. This implies $b M \subset M$ and so $M$ is $b$-closed.

2.8. Let $X, Y \in T, Z \in T_{0}, Z \notin T_{1}, f \in \operatorname{Hom}_{T}(X, Y)$. Let $\phi=\psi$ for each $\phi, \psi$ $\in \operatorname{Hom}_{T}(Y, Z), \phi f=\psi f$. Then $b f(X)=Y$.

Proof. Let $y \in Y-b f(X)$. Then there exist open sets $G_{1}, G_{2}$ of the space $Y$ such that $G_{1} \cap f(X)=G_{2} \cap f(X)$ and $y \in G_{2}-G_{1}$. According to the suppositions mentioned in the assertion there exist $z_{1}, z_{2} \in Z, z_{1} \in \operatorname{cl}\left\{z_{2}\right\}, z_{2} \notin \operatorname{cl}\left\{z_{1}\right\}$. For $i=1,2$ put

$$
\begin{aligned}
\phi_{i}(t) & =z_{1} & & \text { for } t \in Y-G_{i}, \\
& =z_{2} & & \text { for } t \in G_{i} .
\end{aligned}
$$

Then $\phi_{i} \in \operatorname{Hom}_{T}(Y, Z), \phi_{1}(y) \neq \phi_{2}(y), \phi_{1} f=\phi_{2} f$, which is a contradiction.

\section{3. $b$-embedding reflector.}

3.1. Definition. Let $F: T \rightarrow P$ be a reflector. $F$ is called a $b$-embedding reflector iff $P \subset T_{0}$ and if $e_{X}(X)$ is a $b$-dense subset of $F(X)$ for all $X \in T$.

$\left({ }^{3}\right) i_{X}$ denotes the identity on $X$. 
3.2. Definition. A topological property $P$ is called a $b$-embedding iff there exists a $b$-embedding reflector $F: T \rightarrow P$.

3.3. Definition. A topological property $P$ is $b$-closed-hereditary if $A \in P$ whenever $A$ is a $b$-closed subspace of some $Q \in P$.

3.4. TheOREM. A topological property $P$ is b-embedding iff $P$ is productive, b-closed-hereditary and $P \subset T_{0}$.

Proof. Let $B$ denote the category of all ordered triples $(A, e, X)$, where $A$ is a set, $X \in T_{0}, e$ is a mapping from $B$ into $X$ and $b e(A)=X$. A morphism $g:(A, e, X)$ $\rightarrow(C, f, Y)$ is a mapping $g$ from $A$ into $C$ for which there exists a continuous mapping $\bar{g} \in \operatorname{Hom}_{T}(X, Y)$ such that $\bar{g} e=f g$.

Let $S$ be the category of all sets and mappings, $K$ be the functor $B \rightarrow S$ for which $K(A, e, X)=A$ and $K(g)=g$. By means of the assertions of $\S 2$ we prove as in the third section of Kennison's paper [4] that $K$ is a pullback stripping functor, whence we prove this theorem in the same manner as Theorems $C$ and D are proved in [4].

REMARK. This theorem can be proved easily also by means of the Theorem 3 in Hušek's paper [2] and by means of the assertion of $\S 2$ of this paper.

3.5. THEOREM. Let $P$ be a topological property, which is a reflective subcategory of $T$. If $P \subset T_{0}, P \notin T_{1}$, then $P$ is $b$-embedding. If $P \notin T_{0}$, then $P$ is simple.

Proof. According to $1.1 P$ is productive.

I. Let $P \subset T_{0}, \mathrm{P}$ non $\subset T_{1}$. Let $F$ be a reflector: $T \rightarrow P$. Let $X \in P, Y \subset X, b Y=Y$, $i$ denote the identical embedding $Y$ into $X$. Then there exists one morphism $j \in \operatorname{Hom}_{T}(F(Y), X)$ such that $j e_{Y}=i$. Thus it follows that $e_{Y}$ is a homeomorphism from $Y$ into $F(Y)$. According to $2.8 b e_{Y}(Y)=F(Y)$ holds; from 2.5 we get $j F(Y)=Y$ and from 2.6 it follows $e_{Y}(Y)=F(Y)$; hence $P$ is a $b$-embedding.

II. Let $P$ non $\subset T_{0}$. Then there exist $Z \in T, z_{1}, z_{2} \in Z, z_{1} \neq z_{2}, z_{1} \in \operatorname{cl}\left\{z_{2}\right\}$, $z_{2} \in \operatorname{cl}\left\{z_{1}\right\}$. Then each mapping $f$ of a space $X$ into $Z$ with the property $f(X)$ $\subset\left\{z_{1}, z_{2}\right\}$ is continuous; thus it follows easily that $P$ is simple.

4. Remark. Let $Q=\left\{q_{1} \neq q_{2}\right\}$ denote a two-element set and $U=\left\{\left\{q_{1}\right\}, Q, \varnothing\right\}$. Then $(Q, U) \in T_{0}$. Let $P^{\prime}$ be the class of all topological spaces of the type $\Pi Q_{\iota}$ $(\iota \in \mathscr{I})$, where $\mathscr{I}$ is a nonvoid set and $Q_{\iota}=(Q, U)$ for each $\iota \in \mathscr{I}$. Let $P$ (resp. $P^{*}$ ) be the class of all $b$-closed (resp. closed) subspaces of a space from $P^{\prime}$ and of all spaces homeomorphic to these spaces.

4.1. $P\left(\right.$ resp. $\left.P^{*}\right)$ is a topological property, which is productive and b-closed (resp. closed) hereditary.

Proof. We can see clearly that $P$ is a topological property, which is $b$-closed hereditary. From 2.7 it follows that $P$ is productive. We can prove easily the assertion about the class $P^{*}$.

4.2. Let $\varnothing \neq X$ be a subspace of $Y \in T_{0}$, let $Z \in P^{\prime}, f \in \operatorname{Hom}_{T}(X, Z)$. Then there exists at least one $g \in \operatorname{Hom}_{T}(Y, Z), g \mid X=f$. 
Proof. I. Let $Z=(Q, U)$. Then $f^{-1}\left(q_{1}\right)$ is an open set of $X$; therefore there exists an open set $G$ of $Y$ such that $G \cap X=f^{-1}\left(q_{1}\right)$. Put

$$
\begin{array}{rlrl}
g(y)=q_{1} & & \text { for } y \in G, \\
& =q_{2} & & \text { for } y \in Y-G .
\end{array}
$$

Then $g \in \operatorname{Hom}_{T}(Y, Z)$ and $g \mid X=f$.

II. Let $Z=\prod Q_{\iota}(\iota \in \mathscr{I} \neq \varnothing), Q_{\iota}=(Q, U), p_{\iota}$ be the projection $Z \rightarrow Q_{\imath}$. According to I for each $\iota \in \mathscr{I}$ there exists $g_{\iota} \in \operatorname{Hom}_{T}\left(Y, Q_{\iota}\right), g_{\iota} \mid X=p_{\iota} f$. Then the mapping $g$ defined $p_{\imath} g=g_{\imath}$ has the property sought.

4.3. Let $\varnothing \neq X \in P^{*}$. Then there exists $x_{0} \in X$ such that $x_{0}$ has only one neighborhood $X$.

Proof. If $X \subset \prod Q_{\iota}(\iota \in \mathscr{I} \neq \varnothing), Q_{\iota}=(Q, U)$ and $X$ is closed, then we shall put $x_{0}(\iota)=q_{2}$ for each $\iota \in \mathscr{I}$. Then $x_{0} \in X$ and only one neighborhood of $x_{0}$ is $X$.

4.4. Let $R=\prod_{n=1}^{\infty} Q_{n}$, where $Q_{n}=(Q, U), S=R-\left\{r_{0}\right\}$, where $r_{0}(n)=q_{1}$ for each positive integer $n$. Since each nonvoid open set of $R$ contains the point $r_{0}$, it holds $b_{R} S=R$. From 4.2 and $2.5 R$ is the reflection of $S$ in $P$. According to the Theorem $3.4 P$ is the reflective full (replete) subcategory of $T$ and $P$ is neither simple nor identifying, nor embedding, which are types mentioned in Kennison's paper [4].

Let $S^{*}=\left\{x \in R \mid\right.$ there exists a positive integer $n$ such that $\left.x(n)=q_{1}\right\}$. $S^{*}$ is an open set of $R$ and according to Corollary of $2.2 b_{R} S^{*}=S^{*}$. From $4.3 S^{*} \notin P^{*}$. Therefore according to the Theorem $3.5 P^{*}$ is not a reflective subcategory of $T$. From this the $b$-closed-hereditary property in Theorem 3.4 cannot be replaced by the closed-hereditary property.

\section{REFERENCES}

1. E. Cech, Topological spaces, Academia Prague, 1966.

2. M. Hušek, Remarks on reflections, Comment. Math. Univ. Carolinae 7 (1966), 249-259.

3. J. Kelley, General topology, Van Nostrand, Princeton, N. J., 1955.

4. J. F. Kennison, Reflective functors in general topology and elsewhere, Trans. Amer. Math. Soc. 118 (1965), 303-315.

5. S. Baron, $A$ note on epi in $T_{0}$, Canad. Math. Bull. (to appear).

UNIVERSITY OF BRNO,

BRno, Czechoslovakia 